\title{
OVERLAPPING LQ CONTROL OF DISCRETE-TIME TIME-VARYING SYSTEMS
}

\author{
Lubomír Bakule*, José Rodellar** and \\ Josep M. Rossell *.
}

- Institute of Information Theory and Automation, Academy of Sciences of the Czech Republic, 18208 Prague 8, Czech Republic (bakule@utia.cas.cz).

** Department of Applied Mathematics III, Technical University of Catalonia (UPC), Campus Nord, C-2, 08034-Barcelona, Spain (jose.rodellar@upc.es).

-.- Department of Applied Mathematics III, Technical University of Catalonia (UPC), (18240-Manresa, Spain

(josep.maria.rossell@upc.es).

\begin{abstract}
Overlapping decentralized linear quadratic control design problem for discrete-time time-varying systems is solved by using generalized selection of complementary matrices. Explicit conditions on the structure of complementary matrices are derived, including conditions on contractability of controllers. Systematic procedure for their selection is given. Copvright 2000 IFAC
\end{abstract}

Keywords: Decentralized control, large-scale systems, linear systems, discrete-time systems, time-varying systems, overlapping decompositions, optimal control.

\section{INTRODUCTION}

The inclusion principle has been developed by Siljak and his co-workers (Ikeda et al. 1981),(Ikeda ct al. 1984a),(Siljak 1991). It defines a framework for two dynamic systems with different dimensions, in which solutions of the system with larger dimension includes solutions of the system with smaller dimension. The conditions on complementary matrices, which are presented there, have implicit character in the sense that it is difficult to select their specific values. In fact only two particular forms of aggregations and restrictions have been used for the control design and numeriral romputations. Recently, a generalizer procedure for selection of complementary matrices has

1 This work was supported in part by the MLC under (irant 1) GiESIC:SABI999-0114, by the ASC:R under Grant A2075802, by the CICYT under P'roject TAI'999-1079.CO3.

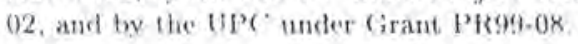

been developed as a mean to overcome this drawback (Bakule et al. 2000a), (Bakule et al. 2000b), (Rossell 1998). However, these results concern continuous-time linear time invariant (LTI) systems. The only results for linear time-varying (LTV) systems are given in (Ikeda et al. 1984b). Concerning discrete-time LTI systems, only multirate control systems have been considered in (Ito 1998) because the extension from continuoustime LTI systems to its discrete-time single rate counterpart is straightforward. However, this extension is not straightforward when considering LTV systems within the inclusion principle.

In this paper, it is shown how to extend the strategy of generalized selection of complementary matrices for the decentralized linear quadratic optimal control design of discrete-time LTV systems. 


\section{PROBLEM FORMULATION}

Consider the optimal control problems as follows:

$$
\begin{aligned}
& \min _{u(k)} J\left(x\left(k_{0}\right), u(k)\right)=x^{T}\left(k_{f}\right) \Pi x\left(k_{f}\right)+ \\
& +\sum_{k=k_{0}}^{k_{f}-1}\left[x^{T}(k) Q^{*}(k) x(k)+u^{T}(k) R^{*}(k) u(k)\right], \\
& \text { s.t. S: } \quad x(k+1)=A(k) x(k)+B(k) u(k)
\end{aligned}
$$

and

$$
\begin{aligned}
& \min _{\tilde{u}(k)} \tilde{J}\left(\tilde{x}\left(k_{0}\right), \bar{u}(k)\right)=\tilde{x}^{T}\left(k_{f}\right) \tilde{\Pi} \tilde{x}\left(k_{f}\right)+ \\
& +\sum_{k=k_{0}}^{k_{1}-1}\left[\tilde{x}^{T}(k) \tilde{Q}^{*}(k) \bar{x}(k)+\tilde{u}^{T}(k) \tilde{R}^{*}(k) \bar{u}(k)\right], \\
& \text { s.t. } \tilde{\mathbf{S}}: \quad \tilde{x}(k+1)=\tilde{A}(k) \tilde{x}(k)+\tilde{B}(k) \tilde{u}(k),
\end{aligned}
$$

where $k_{0}$ is the initial time, $k$, is the final time and integers $k \in\left[k_{0}, k_{0}+1, \ldots, k_{f}\right]$. The vectors $x(k) \in \mathbb{R}^{n}, u(k) \in \mathbb{R}^{m}$ and $\bar{x}(k) \in \mathbb{R}^{\tilde{n}}, \bar{u}(k) \in \mathbb{R}^{\tilde{m}}$ are the states and inputs of $\mathbf{S}$ and $\tilde{\mathbf{S}}$ at time $k$ for $k \in\left[k_{0}, k_{\jmath}\right]$, respectively, $A(k), B(k), Q^{*}(k)$, $R^{*}(k)$ and $\bar{A}(k), \tilde{B}(k), \tilde{Q}^{*}(k), \tilde{R}^{*}(k)$ are matrices of appropriate dimensions satisfying standard assumptions on the LQ design. Suppose $n \leqslant \bar{n}$. $x(k)=x\left(k ; x\left(k_{0}\right), u(k)\right), \tilde{x}(k)=\tilde{x}\left(k ; \tilde{x}\left(k_{0}\right), \tilde{u}(k)\right)$ denote the solutions of (1), (2) for given inputs $u(k), \tilde{u}(k)$, respectively. Given an initial time $k_{0}$, an initial state $x\left(k_{0}\right)$ and an input signal $u(k)$ defined for all $k \in\left[k_{0}, k_{f}\right]$, it is well known that

$$
\begin{aligned}
& x(k)=\Phi\left(k, k_{0}\right) x\left(k_{0}\right)+\sum_{j=k_{0}}^{k-1} \Phi(k, j+1) B(j) u(j), \\
& \tilde{x}(k)=\tilde{\Phi}\left(k, k_{0}\right) \tilde{x}\left(k_{0}\right)+\sum_{j=k_{0}}^{k-1} \tilde{\Phi}(k, j+1) \tilde{B}(j) \tilde{u}(j)
\end{aligned}
$$

are the unique solutions of the systems (1) and (2), respectively, $\Phi, \tilde{\Phi}$ are discrete-time transition matrices (Rugh 1996). Suppose the sum is zero if $k=k_{o}$. Specifically, and only for the ordering of arguments corresponding to forward iteration, let us denote

$$
\Phi(k, j)=A(k-1) A(k-2) \cdots A(j), k \geqslant j
$$

by adopting the convention that an empty product is the identity, i.e. $\Phi(k, j)=I$ if $k=j$.

$\mathrm{S}$ and $\tilde{\mathbf{S}}$ are related by the transformations $\bar{x}(k)=$ $V x(k), x(k)=U \bar{x}(k), \tilde{u}(k)=R u(k), u(k)=$ $Q \tilde{u}(k)$, where $V, U, R$ and $Q$ are constant fullrank matrices of appropriate dimensions.

Definition 1. We say that a pair $\left(\tilde{\mathbf{S}}_{1}, \tilde{J}\right)$ includes a pair $(\mathbf{S}, J)$, that is $(\overline{\mathbf{S}}, \bar{J}) \supset(\mathbf{S}, J)$, if there exist a quadruplet of constant matrices $(U, V, Q, R)$ such that $U V=I_{n}, Q R=I_{m}$ and for any initial state $x\left(k_{0}\right)$ and any fixed $u(k)$ of $\mathbf{S}, x\left(k ; x\left(k_{0}\right), u(k)\right)=$
$U \tilde{x}\left(k ; V x\left(k_{0}\right), R u(k)\right)$ for all $k \in\left[k_{0}, k_{f}\right]$; and $J\left(x\left(k_{0}\right), u(k)\right)=\tilde{J}\left(V x\left(k_{0}\right), R u(k)\right)$.

Definition 2. If $(\tilde{\mathbf{S}}, \bar{J}) \supset(\mathbf{S}, J)$, then $(\tilde{\mathbf{S}}, \tilde{J})$ is called an expansion of $(\mathbf{S}, J)$ and $(\mathbf{S}, J)$ is a contraction of $(\tilde{\mathbf{S}}, \tilde{J})$.

Definition 3. A control law $\bar{u}(k)=-\tilde{K}(k) \bar{x}(k)$ for $\tilde{\mathbf{S}}$ is contractible to the control law $u(k)=$ $-K(k) x(k)$ for $\mathbf{S}$ if the choice $\bar{x}\left(k_{0}\right)=V x\left(k_{0}\right)$ and $\bar{u}(k)=R u(k)$ implies $K(k) x\left(k ; x\left(k_{0}\right), u(k)\right)=$ $Q \tilde{K}(k) \tilde{x}\left(k ; V x\left(k_{0}\right), R u(k)\right)$ for all $k \in\left[k_{0}, k_{f}\right]$, any initial state $x\left(k_{0}\right)$ and any fixed input $u(k)$ of $\mathbf{S}$.

Suppose given pairs of matrices $(U, V)$ and $(Q, R)$. Then the matrices $\tilde{A}(k), \tilde{B}(k), \bar{\Pi}, \tilde{Q}^{*}(k), \tilde{R}^{*}(k)$ and $\bar{K}(k)$ can be described as

$$
\begin{aligned}
\tilde{A}(k) & =V A(k) U+M(k), \\
\tilde{B}(k) & =V B(k) Q+N(k), \\
\tilde{\Pi} & =U^{T} \Pi U+M_{\Pi,} \\
\tilde{Q}^{*}(k) & =U^{T} Q^{*}(k) U+M_{Q^{*}}(k), \\
\tilde{R}^{*}(k) & =Q^{T} R^{*}(k) Q+N_{R^{*}}(k), \\
\tilde{K}(k) & =R K(k) U+F(k),
\end{aligned}
$$

where $M(k), N(k), M_{\Pi}, M_{Q^{*}}(k), N_{R^{*}}(k)$ and $F(k)$ are complementary matrices.

The problem. The specific goals are as follows: (i) To derive explicit conditions on complementary matrices satisfying $(\tilde{\mathbf{S}}, \tilde{J}) \supset(\mathbf{S}, J)$ for discrete LTV systems including contractibility conditions; (ii) To present a systematic procedure for their selection.

\section{MAIN RESULTS}

Agreement: Straightforward proofs are omitted.

Theorem 4. Consider S, $\overline{\mathbf{S}}$ given by (1), (2), respectively. $\tilde{\mathbf{S}} \supset \mathbf{S}$ if and only if

$$
\begin{array}{r}
U\left[\tilde{\Phi}\left(k, k_{0}\right) \tilde{x}\left(k_{0}\right)+\sum_{j=k_{0}}^{k-1} \tilde{\Phi}(k, j+1) \tilde{B}(j) \tilde{u}(j)\right]= \\
=\Phi\left(k, k_{0}\right) x\left(k_{0}\right)+\sum_{j=k_{0}}^{k-1} \Phi(k, j+1) B(j) u(j)
\end{array}
$$

for all $k \in\left[k_{0}, k_{l}\right]$.

We must impose some conditions on complementary matrices by $(5)$ to satisfy $(\tilde{\mathbf{S}}, \tilde{J}) \supset(\mathbf{S}, J)$. Define for integers $r, s \in\left[k_{0}, k_{f}\right]$ and any square matrix $\mathcal{M}$ the matrix product $\mathcal{M}[r, s]$ as follows:

$$
\begin{aligned}
& \mathcal{M}[r, s]=\mathcal{M}(r) \mathcal{M}(r-1) \cdots \mathcal{M}(s), r>s \\
& \mathcal{M}[r, s]=\mathcal{M}(r), r=s .
\end{aligned}
$$


$\mathcal{M}[r, s]$ is undefined for $r<s$. Now, we are ready to formulate the inclusion principle for discretetime LTV systems in terms of complementary matrices.

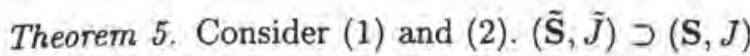
if and only if

$$
\begin{aligned}
U M[r, s] V & =0, & U N(r) R & =0, \\
U M[p, q] N(q-1) R & =0, & V^{T} M_{\mathrm{n}} V & =0, \\
V^{T} M_{Q^{*}}(k) V & =0, & R^{T} N_{R^{*}}(k) R & =0
\end{aligned}
$$

hold for all fixed $k \in\left[k_{0}, k_{f}\right]$, for all $r, s$ such that $k_{0} \leqslant s \leqslant r \leqslant k-1$ and all $p, q$ such that $k_{\mathrm{o}}+1 \leqslant q \leqslant p \leqslant k-1$.

Proof. By using Definition 1 and Theorem 4, we get: 1) $U \tilde{\Phi}\left(k, k_{0}\right) V x\left(k_{0}\right)=\Phi\left(k, k_{0}\right) x\left(k_{0}\right)$ and further 2) $\sum_{j=k_{0}}^{k-1} U \tilde{\Phi}(k, j+1) \tilde{B}(j) \bar{u}(j)=$ $\sum_{j=k_{0}}^{k-1} \Phi(k, j+1) B(j) u(j)$ for all $k \in\left[k_{0}, k_{f}\right]$. If $\left.k=k_{0}, 1\right)$ and 2) hold directly. By using (5) and from 1) for all fixed $k \in\left[k_{0}+1, k_{f}\right]$, we obtain $U M[r, s] V=0$ for all $r \in\left[k_{0}, k-1\right]$ and all $s \in\left[k_{0}, r\right]$, i.e. $U M[r, s] V=0$ for all $r, s$ such that $k_{0} \leqslant s \leqslant r \leqslant k-1$. By using (5), for all fixed $\left.k \in\left[k_{0}+1, k_{\jmath}\right], 2\right)$ is equivalent to $U N(r) R=0$ and $U M[p, q] N(q-1) R=0$, for all $r \in\left[k_{0}, k-1\right]$ and all $p, q$ such that $k_{0}+1 \leqslant q \leqslant p \leqslant k-1$. The remaining conditions can be obtained easily when considering $J\left(x\left(k_{0}\right), u(k)\right)=\bar{J}\left(V x\left(k_{0}\right), R u(k)\right)$.

The following theorems give the conditions on complementary matrices to satisfy Definition 3.

Theorem 6. A control law $\tilde{u}(k)=-\tilde{K}(k) \tilde{x}(k)$ for $\overline{\mathrm{S}}$ is contractible to the control law $u(k)=$ $-K(k) x(k)$ for $\mathbf{S}$ if and only if

$$
\begin{aligned}
Q F(k) & {\left[\tilde{\Phi}\left(k, k_{0}\right) V x\left(k_{0}\right)+\right.} \\
& \left.+\sum_{j=k_{0}}^{k-1} \tilde{\Phi}(k, j+1)+\tilde{B}(j) \tilde{u}(j)\right]=0
\end{aligned}
$$

hold for all fixed $k \in\left[k_{0}, k_{f}\right]$.

Theorem 7. A control law $\tilde{u}(k)=-\tilde{K}(k) \tilde{x}(k)$ for $\tilde{\mathbf{S}}$ is contractible to the control law $u(k)=$ $-K(k) x(k)$ for $\mathbf{S}$ if

$$
\begin{aligned}
Q F(k) V & =0, \\
Q F(k) M[k-1, r] V & =0, \\
Q F(k) N(k-1) R & =0, \\
Q F(k) M[k-1, p] N(p-1) R & =0
\end{aligned}
$$

hold for all fixed $k \in\left[k_{0}, k_{f}\right]$, all $r \in\left[k_{0}, k-1\right]$ and all $p \in\left[k_{0}+1, k-1\right]$.

Proof. By using $\tilde{B}(k)=V B(k) Q+N(k)$ of $(5)$, Theorem 6 is equivalent to: 1) $Q F(k) \tilde{\Phi}\left(k, k_{0}\right) V=$
0, 2) $Q F(k) \sum_{j=k_{0}}^{k-1} \tilde{\Phi}(k, j+1) V B(j)=0$, and 3) $Q F(k) \sum_{j=k_{0}}^{k-1} \tilde{\Phi}(k, j+1) N(j) R=0$, for all fixed $k \in\left[k_{0}, k_{f}\right]$. If $k=k_{0}$, only the relation $Q F\left(k_{0}\right) V=0$ is defined. If $Q F(k) V=0$ and $Q F(k) M[k-1, r] V=0$, for all fixed $k \in\left[k_{0}+1, k\right.$, $]$ and all $r \in\left[k_{0}, k-1\right]$, then 1) holds. 1) implies 2). If $Q F(k) N(k-1) R=0$ and $Q F(k) M[k-$ $1, p] N(p-1) R=0$ for all $p \in\left[k_{0}+1, k-1\right]$, then 3) holds.

\subsection{Expansion-contraction process}

Change of basis. The change of basis in the expansion-contraction process introduced in (Ikeda et al. 1984a),(Siljak 1991) represents $\tilde{\mathrm{S}}$ in a canonical form. Since the inclusion principle does not depend on the specific basis used in the state, input and output spaces, we may introduce convenient changes of basis in $\tilde{\mathbf{S}}$ for a prespecified purpose (Bakule et al. 2000b), (Rossell 1998). The expansion-contraction process between $\mathbf{S}$ and $\tilde{\mathbf{S}}$ can be illustrated in the form

$$
\begin{aligned}
& \mathrm{S} \rightarrow \overline{\mathrm{s}} \longrightarrow \overline{\overline{\mathrm{S}}} \rightarrow \overline{\mathrm{S}} \rightarrow \mathrm{S} \text {. } \\
& \mathbb{R}^{\mathrm{n}} \stackrel{V}{\rightarrow} \mathbb{R}^{\bar{n}} \stackrel{T_{A}^{-1}}{\longrightarrow} \overline{\mathbb{R}}^{\bar{n}} \stackrel{T_{A}}{\longrightarrow} \mathbb{R}^{\mathrm{n}} \stackrel{U}{\rightarrow} \mathbb{R}^{n}, \\
& \mathbb{R}^{m} \stackrel{R}{\longrightarrow} \mathbb{R}^{\dot{m}} \stackrel{T_{B}^{-1}}{\longrightarrow} \overline{\mathbb{R}}^{\dot{m}} \stackrel{T_{B}}{\longrightarrow} \mathbb{R}^{\dot{m}} \stackrel{Q}{\longrightarrow} \mathbb{R}^{m},
\end{aligned}
$$

where $\overline{\overline{\mathbf{S}}}$ denotes the expanded system in a new bases. Given $V$ and $R$, we define $U=$ $\left(V^{T} V\right)^{-1} V^{T}, Q=\left(R^{T} R\right)^{-1} R^{T}$ as their pseudoinverses, respectively. Let us consider

$$
T_{A}=\left(V W_{A}\right), \quad T_{B}=\left(R W_{B}\right),
$$

where $W_{A}, W_{B}$ are chosen such that $\operatorname{Im} W_{A}=$ Ker $U, \operatorname{Im} W_{B}=\operatorname{Ker} Q$. By using these transformations, the conditions $\bar{U} \bar{V}=I_{n}, \bar{V} \bar{U}=\left(\begin{array}{cc}I_{n} & 0 \\ 0 & 0\end{array}\right)$ and $\bar{Q} \bar{R}=I_{m}, \bar{R} \bar{Q}=\left(\begin{array}{cc}I_{m} & 0 \\ 0 & 0\end{array}\right)$ can be easily verified, where $\bar{V}=T_{A}^{-1} V=\left(\begin{array}{c}I_{n} \\ 0\end{array}\right), \bar{U}=U T_{A}=\left(\begin{array}{ll}I_{n} & 0\end{array}\right)$ and $\bar{R}=T_{B}^{-1} R=\left(\begin{array}{c}I_{m} \\ 0\end{array}\right), \bar{Q}=Q T_{B}=\left(\begin{array}{ll}I_{m} & 0\end{array}\right)$. Note that the motivating factor for defining $T_{A}$ and $T_{B}$ by (12) is the fulfillment of these conditions. They play a crucial role in deriving explicit block structured complementary matrices (with zero blocks) including a general strategy for their selection.

Expansion-contraction in the new basis. Consider the system $\mathbf{S}$ partitioned as follows:

$$
\begin{gathered}
\left(\begin{array}{l}
x_{1}(k+1) \\
x_{2}(k+1) \\
x_{3}(k+1)
\end{array}\right)=\left(\begin{array}{l:l:c}
A_{11}(k) & A_{12}(k) & A_{13}(k) \\
A_{21}(k) & A_{22}(k) & A_{22}(k) \\
\hdashline A_{31}(k) & A_{32}(k) & A_{33}(k)
\end{array}\right)\left(\begin{array}{l}
x_{1}(k) \\
x_{2}(k) \\
x_{3}(k)
\end{array}\right) \\
+\left(\begin{array}{ll:l}
B_{11}(k) & B_{12}(k) & B_{13}(k) \\
B_{21}(k) & B_{22}(k) & B_{21}(k) \\
\hdashline B_{31}(k) & B_{32}(k) & B_{39}(k)
\end{array}\right)\left(\begin{array}{l}
u_{1}(k) \\
u_{2}(k) \\
u_{3}(k)
\end{array}\right), \quad(13)
\end{gathered}
$$

where $A_{i i}(k), B_{i i}(k), i=1,2,3$, are $n_{i} \times n_{i}, n_{i} \times$ $m_{i}$ matrices, respectively. This structure has been 
adopted as a prototype structure for overlapping decompositions (Ikeda et al. 1981),(Ikeda et al. 1984a),(Ikeda et al. 1984b),(Siljak 1991).

Define $(\tilde{\overline{\mathrm{S}}}, \tilde{\bar{J}})$ as follows:

$$
\begin{aligned}
& \min _{\substack{\tilde{u}(k)\\
}} \overline{\bar{J}}\left(\tilde{\bar{x}}\left(k_{0}\right), \tilde{\tilde{u}}(k)\right)=\tilde{\bar{x}}^{T}\left(k_{f}\right) \overline{\bar{\Pi}} \tilde{\bar{x}}\left(k_{f}\right)+ \\
& +\sum_{k=k_{0}}^{k_{f}-1}\left[\overline{\bar{x}}^{T}(k) \tilde{\bar{Q}}^{*}(k) \tilde{\bar{x}}(k)+\tilde{\bar{u}}^{T}(k) \tilde{\tilde{R}}^{*}(k) \overline{\bar{u}}(k)\right] \text {, } \\
& \text { s.t. } \overline{\overline{\mathrm{S}}}: \dot{\bar{x}}(k+1)=\tilde{\bar{A}}(k) \tilde{\bar{x}}(k)+\dot{\bar{B}}(k) \tilde{\tilde{u}}(k)
\end{aligned}
$$

for all $k \in\left[k_{0}, k_{f}\right]$, where $\tilde{\bar{A}}(k), \tilde{\bar{B}}(k), \overline{\bar{\Pi}}, \tilde{\bar{Q}}^{*}(k)$ and $\tilde{\tilde{R}}^{*}(k)$ are matrices of appropriate dimensions. The vectors $\tilde{\tilde{\bar{x}}}(k)$ and $\tilde{\tilde{u}}(k)$ are defined as $\tilde{\bar{x}}(k)=$ $T_{A}^{-1} V x(k)=\bar{V} x(k), \tilde{\tilde{u}}(k)=T_{B}^{-1} R u(k)=\bar{R} u(k)$. Now, the relations between $\overline{\overline{\mathbf{S}}}$ and $\mathbf{S}$ are defined as $\tilde{\bar{A}}(k)=\bar{V} A(k) \bar{U}+\bar{M}(k), \quad \overline{\bar{B}}(k)=\bar{V} B(k) \bar{Q}+$ $\bar{N}(k), \overline{\bar{\Pi}}=\bar{U}^{T} \Pi \bar{U}+\bar{M}_{\Pi}, \overline{\bar{Q}}^{*}(k)=\bar{U}^{T} Q^{*}(k) \bar{U}+$ $\bar{M}_{Q^{*}}(k), \tilde{\tilde{R}}^{*}(k)=\bar{Q}^{T} R^{*}(k) \bar{Q}+\bar{N}_{R^{*}}(k)$, where new complementary matrices are

$$
\begin{aligned}
\bar{M}(k) & =T_{A}^{-1} M(k) T_{A}, \\
\bar{N}(k) & =T_{A}^{-1} N(k) T_{B}, \\
\bar{M}_{\Pi} & =T_{A}^{T} M_{\Pi} T_{A}, \\
\bar{M}_{Q} \cdot(k) & =T_{A}^{T} M_{Q} \cdot(k) T_{A}, \\
\bar{N}_{R^{*}}(k) & =T_{B}^{T} N_{R^{*}}(k) T_{B} .
\end{aligned}
$$

First, we analyze the structure of the matrices $\bar{M}(k), \bar{N}(k), \bar{M}_{\Pi}, \bar{M}_{Q \cdot} \cdot(k)$ and $\bar{N}_{R} \cdot(k)$ in the expanded system. Consider the complementary matrices of $\tilde{\mathbf{S}}$ in the form $M(k)=M_{i j}(k)$, $N(k)=N_{i j}(k), M_{\Pi}=M_{\Pi_{i j}}, M_{Q}(k)=$ $M_{Q_{i j}^{*}}(k), N_{R^{*}}(k)=N_{R_{i j}^{*}}(k)$ for $i, j=1, \ldots, 4$, where $M_{\Pi_{i j}}=M_{\Pi_{j i}}^{T}, M_{Q_{i j}^{*}}(k)=M_{Q_{j i}^{*}}^{T}(k)$, $N_{R_{i j}^{*}}(k)=N_{R_{j i}^{*}}^{T}(k)$ and each matrix dimensions correspond to (13). Consider the matrices $\bar{M}(k)=$ $\left(\begin{array}{ll}\bar{M}_{11}(k) & \bar{M}_{12}(k) \\ \bar{M}_{21}(k) & \bar{M}_{22}(k)\end{array}\right), \bar{N}(k)=\left(\begin{array}{ll}\bar{N}_{11}(k) & \bar{N}_{12}(k) \\ \bar{N}_{21}(k) & \bar{N}_{22}(k)\end{array}\right), \quad \bar{M}_{\Pi}=$ $\left(\begin{array}{ll}\bar{M}_{\mathrm{n}_{11}} & \bar{M}_{\mathrm{n}_{12}} \\ \bar{M}_{\mathrm{n}_{12}}^{T} & \bar{M}_{\mathrm{n}_{22}}\end{array}\right), \quad \bar{M}_{Q \cdot}(k)=\left(\begin{array}{ll}\bar{M}_{Q_{\mathrm{i}_{1}}}(k) & \bar{M}_{Q_{Q_{2}}}(k) \\ \bar{M}_{Q_{\mathrm{i}_{2}}}^{T}(k) & \bar{M}_{Q_{22}^{*}}(k)\end{array}\right)$, $\bar{N}_{R^{*}}(k)=\left(\begin{array}{lll}\bar{N}_{R_{11}^{*}}(k) & \bar{N}_{R_{12}^{*}}(k) \\ \bar{N}_{R_{12}}^{\tau}(k) & \bar{N}_{R_{22}^{*}}(k)\end{array}\right)$, where $\bar{M}_{11}(k)$, $\bar{M}_{22}(k)$ are $n \times n, n_{2} \times n_{2}$ matrices, respectively. $\bar{N}_{11}(k), \bar{N}_{22}(k)$ are $n \times m, n_{2} \times m_{2}$ matrices, respectively. $\bar{M}_{\Pi_{11}}, \bar{M}_{\Pi_{22}}$ are $n \times n, n_{2} \times n_{2}$ matrices, respectively. $\bar{M}_{Q_{11}^{*}}(k), \bar{M}_{Q_{22}^{*}}(k)$ are $n \times n, n_{2} \times n_{2}$ matrices, respectively. $\bar{N}_{R_{11}^{*}}(k), \bar{N}_{R_{22}^{*}}(k)$ are $m \times m$, $m_{2} \times m_{2}$ matrices, respectively. We need to know the form of these submatrices. This is presented by the following propositions.

Proposition 8. Consider $\mathbf{S}$ and $\tilde{\overline{\mathbf{S}}}$ given by (1) and (14) satisfying $\overline{\tilde{\mathbf{S}}} \supset \mathrm{S}$, respectively. Then $\bar{M}(r)=\left(\begin{array}{cc}0 & \bar{M}_{12}(r) \\ \bar{M}_{21}(r) & \bar{M}_{22}(r)\end{array}\right)$, where $(0)$ denotes a null matrix of order $n$ and the other blocks satisfy $\bar{M}_{12}(p) \bar{M}_{21}(p-1)=0$ and $\bar{M}_{12}(p) \bar{M}_{22}[p-$
$1, j] \bar{M}_{21}(j-1)=0$ for all fixed $k \in\left[k_{0}, k_{f}\right]$, all $r \in\left[k_{0}, k-1\right]$, all $p \in\left[k_{0}+1, k-1\right]$ and all $j \in\left[k_{0}+1, p-1\right]$.

Proof. Denote $\bar{M}(k)=\left(\begin{array}{ll}\bar{M}_{11}(k) & \bar{M}_{12}(k) \\ \bar{M}_{21}(k) & \bar{M}_{22}(k)\end{array}\right)$. Consider the condition $\bar{U} \bar{M}[r, s] \bar{V}=0$, for $k_{0} \leqslant s \leqslant r \leqslant k-$ 1 given by Theorem 5 . $\bar{U} \bar{M}\left[k_{0}, k_{0}\right] \bar{V}=0$ implies $\bar{M}_{11}\left(k_{0}\right)=0$ for $k=k_{0}+1$. We obtain $\vec{M}_{11}\left(k_{0}\right)=$ $0, \bar{M}_{11}\left(k_{0}+1\right)=0$ and $\bar{M}_{12}\left(k_{0}+1\right) \bar{M}_{21}\left(k_{0}\right)=0$ for $k=k_{0}+2$. We get $\bar{M}_{11}(r)=0$ together with the conditions $\bar{M}_{12}(p) \bar{M}_{21}(p-1)=0, \bar{M}_{12}(p) \bar{M}_{22}[p-$ $1, j] \bar{M}_{21}(j-1)=0$ for all $r \in\left[k_{0}, k-1\right]$, all $p \in\left[k_{0}+1, k-1\right]$ and all $j \in\left[k_{0}+1, p-1\right]$ when repeating this process.

Proposition 9. Consider $\mathbf{S}$ and $\overline{\overline{\mathbf{S}}}$ given by (1) and (14) satisfying $\overline{\overline{\mathbf{S}}} \supset \mathbf{S}$, respectively. Then $\bar{N}(r)=$ $\left(\begin{array}{cc}0 & \bar{N}_{12}(r) \\ \bar{N}_{21}(r) & \bar{N}_{22}(r)\end{array}\right)$, where $(0)$ is an $n \times m$ matrix and the other blocks satisfy $\bar{M}_{12}(p) \bar{N}_{21}(p-1)=0$ and $\bar{M}_{12}(p) \bar{M}_{22}[p-1, j] \bar{N}_{21}(j-1)=0$ for all fixed $k \in\left[k_{0}, k_{f}\right]$, all $r \in\left[k_{0}, k-1\right]$, all $p \in\left[k_{0}+1, k-1\right]$ and all $j \in\left[k_{0}+1, p-1\right]$.

Theorem 10. Consider $\mathbf{S}$ and $\overline{\overline{\mathbf{S}}}$ given by (1) and (14), respectively. The pair $(\tilde{\overline{\mathbf{S}}}, \tilde{\bar{J}}) \supset(\mathbf{S}, J)$ if $\bar{M}_{\Pi}=\left(\begin{array}{cc}0 & \bar{M}_{\mathrm{n}_{12}} \\ \bar{M}_{\Pi_{12}}^{T} & \bar{M}_{\mathrm{\Pi}_{22}}\end{array}\right), \bar{M}_{Q^{*}}(k)=\left(\begin{array}{cc}0 & \bar{M}_{Q_{i_{2}}}(k) \\ \bar{M}_{Q_{12}}^{T}(k) & \bar{M}_{Q_{22}^{*}}(k)\end{array}\right)$, $\bar{N}_{R^{*}}(k)=\left(\begin{array}{cc}0 & \bar{N}_{R_{12}^{*}}(k) \\ \bar{N}_{R_{12}}^{T}(k) & \bar{N}_{R_{22}^{*}}(k)\end{array}\right)$ and either

$$
\text { a) } \begin{aligned}
\bar{M}(p) & =\left(\begin{array}{cc}
0 & 0 \\
\bar{M}_{21}(p) & \bar{M}_{22}(p)
\end{array}\right), \\
\text { b) } \bar{M}(p) & =\left(\begin{array}{cc}
0 & \bar{N}_{12}(p) \\
\bar{N}_{21}(p) & \bar{N}_{22}(p)
\end{array}\right) \text { or } \\
\bar{M}(p) & =\left(\begin{array}{ll}
0 & \bar{M}_{12}(p) \\
0 & \bar{M}_{22}(p)
\end{array}\right), \\
\bar{N}(p) & =\left(\begin{array}{ll}
0 & \bar{N}_{12}(p) \\
0 & \bar{N}_{22}(p)
\end{array}\right)
\end{aligned}
$$

hold for all fixed $k \in\left[k_{0}, k_{f}\right]$ and all $p \in\left[k_{0}+\right.$ $1, k-1]$.

Contractibility. The idea is to design a control law for $\tilde{\mathbf{S}}$ so that it can be contracted and implemented into $\mathbf{S}$. Now, we want to determine the conditions under which a control law designed for $\overline{\overline{\mathbf{S}}}$ can be contracted into $\mathbf{S}$ in terms of complementary matrices.

Denote matrices appearing in the contractibility process as follows. The complementary matrix $F(k)$ has the form $F(k)=\left(F_{i j}(k)\right), i, j=1, \ldots, 4$, where $F_{11}(k), F_{22}(k), F_{33}(k)$ and $F_{44}(k)$ are $m_{1} \times$ $n_{1}, m_{2} \times n_{2}, m_{2} \times n_{2}$ and $m_{3} \times n_{3}$ matrices, respectively. Define $\bar{F}(k)=\left(\begin{array}{ll}\bar{F}_{11}(k) & \bar{F}_{12}(k) \\ \bar{F}_{21}(k) & \bar{F}_{22}(k)\end{array}\right)$, where $\bar{F}_{11}(k)$ and $\bar{F}_{22}(k)$ are $m \times n$ and $m_{2} \times n_{2}$ matrices, respectively. Similarly, denote the gain matrix $K(k)=\left(K_{i j}(k)\right), i, j=1,2,3$, where $K_{i i}(k)$ are $m_{i} \times n_{i}$ matrices, respectively. The gain matrix 
$\overline{\bar{K}}(k)$ for $\tilde{\overline{\mathbf{S}}}$ has the form $\overline{\bar{K}}(k)=\bar{R} K(k) \vec{U}+$ $\bar{F}(k)$, where $\tilde{\bar{K}}(k)=T_{B}^{-1} \tilde{K}(k) T_{A}$ and $\bar{F}(k)=$ $T_{B}^{-1} F(k) T_{A}$. So far we do not know the form of the complementary matrix $F(k)$ and the corresponding contractibility conditions. The following theorem solves the problem.

Theorem 11. Consider $\mathbf{S}$ and $\overline{\tilde{\mathbf{S}}}$ given by (1) and (14) satisfying $\tilde{\overline{\mathbf{S}}} \supset \mathbf{S}$, respectively. A control law $\tilde{\tilde{u}}(k)=-\bar{K}(k) \tilde{\tilde{x}}(k)$ for $\overline{\tilde{S}}$ is contractible to the control law $u(k)=-K(k) x(k)$ of $\mathbf{S}$ if $\bar{F}(k)=$ $\left(\begin{array}{cc}0 & \bar{F}_{12}(k) \\ \bar{F}_{21}(k) & \bar{F}_{22}(k)\end{array}\right)$ satisfies

$$
\begin{aligned}
\bar{F}_{12}(k) \bar{M}_{21}(k-1) & =0, \\
\bar{F}_{12}(k) \bar{N}_{21}(k-1) & =0, \\
\bar{F}_{12}(k) \bar{M}_{22}[k-1, p] \bar{M}_{21}(p-1) & =0, \\
\bar{F}_{12}(k) \bar{M}_{22}[k-1, p] \bar{N}_{21}(p-1) & =0
\end{aligned}
$$

for all fixed $k \in\left[k_{0}, k_{f}\right]$ and all $p \in\left[k_{0}+1, k-1\right]$.

Proof. Suppose $\bar{F}(k)=\left(\begin{array}{ll}\bar{F}_{11}(k) & \bar{F}_{12}(k) \\ \bar{F}_{21}(k) & F_{22}(k)\end{array}\right)$. Consider the conditions by Theorem 7 . Only the relation $\bar{Q} \bar{F}(k) \bar{V}=0$ is defined for $k=k_{0}$. We obtain $\bar{F}_{11}\left(k_{0}\right)=0 . \bar{Q} \bar{F}(k) \bar{V}=0, \bar{Q} \bar{F}(k) \bar{M}[k-1, r] \bar{V}=0$ and $\bar{Q} \bar{F}(k) \bar{N}(k-1) \bar{R}=0$ give $\bar{F}_{11}\left(k_{0}+1\right)=0$, $\bar{F}_{12}\left(k_{0}+1\right) \bar{M}_{21}\left(k_{0}\right)=0$ and $\bar{F}_{12}\left(k_{0}+1\right) \bar{N}_{21}\left(k_{0}\right)=$ 0 for $k=k_{0}+1$, respectively. We obtain $\bar{F}_{11}(k)=$ $0, \bar{F}_{12}(k) \bar{M}_{21}(k-1)=0, \bar{F}_{12}(k) \bar{N}_{21}(k-1)=0$, $\bar{F}_{12}(k) \bar{M}_{22}[k-1, p] \bar{M}_{21}(p-1)=0, \bar{F}_{12}(k) \bar{M}_{22}[k-$ $1, p] \bar{N}_{21}(p-1)=0$ for all fixed $k \in\left[k_{0}, k_{t}\right]$ and all $p \in\left[k_{0}+1, k-1\right]$ when repeating this process for all $k$.

\subsection{Selection of complementary matrices}

The above results are general, i.e. they do not depend on the selection of the matrices $V$ and $R$. Therefore, they can be applied to any expansioncontraction process. Specific transformation matrices $V$ and $R$ must be selected to expand a given problem (1) when considering the control design. We select the following expansion transformation matrices:

$$
V=\left(\begin{array}{ccc}
I_{n_{1}} & 0 & 0 \\
0 & I_{n_{2}} & 0 \\
0 & I_{n_{2}} & 0 \\
0 & 0 & I_{n_{3}}
\end{array}\right), R=\left(\begin{array}{ccc}
I_{m_{1}} & 0 & 0 \\
0 & I_{m_{2}} & 0 \\
0 & I_{m_{2}} & 0 \\
0 & 0 & I_{m_{3}}
\end{array}\right)
$$

$x_{2}(k)$ and $u_{2}(k)$ appear in a repeated form in $\tilde{x}(k)=\left(x_{1}^{T}(k), x_{2}^{T}(k), x_{2}^{T}(k), x_{3}^{T}(k)\right)^{T}$ and $\tilde{u}(k)=$ $\left(u_{1}^{T}(k), u_{2}^{T}(k), u_{2}^{T}(k), u_{3}^{T}(k)\right)^{T}$ by using (18), respectively. The change of basis results in

$$
T_{A}=\left(\begin{array}{cccc}
I_{n_{1}} & 0 & 0 & 0 \\
0 & I_{n_{2}} & 0 & I_{n_{2}} \\
0 & I_{n_{2}} & 0 & -I_{n_{2}} \\
0 & 0 & I_{n_{3}} & 0
\end{array}\right)
$$

$$
T_{A}^{-1}=\left(\begin{array}{cccc}
I_{n_{1}} & 0 & 0 & 0 \\
0 & \frac{1}{2} I_{n_{2}} & \frac{1}{2} I_{n_{2}} & 0 \\
0 & 0 \\
0 & \frac{1}{2} I_{n_{2}} & -\frac{1}{2} I_{n_{2}} & 0 \\
0 & I_{n_{3}} & 0
\end{array}\right) .
$$

$T_{B}, T_{B}^{-1}$ have an analogous structure. The following theorems present the structure of the complementary matrices $M(k), N(k), M_{\Pi}, M_{Q^{*}}(k)$, $N_{R^{*}}(k)$ and $F(k)$ in the initial bases.

Theorem 12. Consider $\mathbf{S}$ and $\tilde{\mathbf{S}}$ given by (1) and (2), respectively. $\tilde{\mathbf{S}} \supset \mathbf{S}$ if and only if $M(r)$ has the following structure:

$$
\left(\begin{array}{ccrc}
0 & M_{12} & -M_{12} & 0 \\
M_{21} & M_{22} & M_{23} & M_{24} \\
-M_{21} & -\left(M_{22}+M_{23}+M_{33}\right) & M_{33} & -M_{24} \\
0 & M_{42} & -M_{42} & 0
\end{array}\right)(r)
$$

and their blocks satisfy

$$
\begin{aligned}
& \left(\begin{array}{c}
M_{12} \\
M_{23}+M_{33} \\
M_{42}
\end{array}\right)(p)\left(\begin{array}{lll}
M_{21} & M_{22}+M_{23} & M_{24}
\end{array}\right)(p-1)=0, \\
& \left(\begin{array}{c}
M_{12} \\
M_{23}+M_{33} \\
M_{42}
\end{array}\right)(p)\left(M_{22}+M_{33}\right)[p-1, j] \\
& \left(\begin{array}{lll}
M_{21} & M_{22}+M_{23} & M_{24}
\end{array}\right)(j-1)=0, \\
& \left(\begin{array}{c}
M_{12} \\
M_{23}+M_{33} \\
M_{42}
\end{array}\right)(p)\left(\begin{array}{lll}
N_{21} & N_{22}+N_{23} & N_{24}
\end{array}\right)(p-1)=0, \\
& \left(\begin{array}{c}
M_{12} \\
M_{23}+M_{33} \\
M_{42}
\end{array}\right)(p)\left(M_{22}+M_{33}\right)[p-1, j] \\
& \left(\begin{array}{lll}
N_{21} & N_{22}+N_{23} & N_{24}
\end{array}\right)(j-1)=0
\end{aligned}
$$

for all fixed $k \in\left[k_{0}, k,\right]$, all $r \in\left[k_{0}, k-1\right]$, all $p \in\left[k_{0}+1, k-1\right]$ and all $j \in\left[k_{0}+1, p-1\right]$. The matrix $N(r)$ has the same structure as $M(r)$.

Proof. Consider $\bar{M}(r)=T_{A}^{-1} M(r) T_{A}$ given by (15), where $T_{A}$ and $T^{-1}$ are given by (19). From Proposition $8, \vec{M}_{11}(r)=0$. The other matrix blocks $\bar{M}_{i j}(r), i, j=1,2$ can be identified from Proposition 8. Consequently, we obtain the structure of the complementary matrix $M(r)$ given by $(20)$. An analogous procedure holds for the matrix $N(r)$ when applying Proposition 9. Now, we get (21) when imposing the conditions $\bar{M}_{12}(p) \bar{M}_{21}(p-1)=0, \bar{M}_{12}(p) \bar{M}_{22}[p-1, j] \bar{M}_{21}(j-$ 1) $=0, \bar{M}_{12}(p) \bar{N}_{21}(p-1)=0$ and $\bar{M}_{12}(p) \bar{M}_{22}[p-$ $1, j] \bar{N}_{21}(j-1)=0$ for all fixed $k \in\left[k_{0}, k_{f}\right]$, all $r \in\left[k_{0}, k-1\right]$, all $p \in\left[k_{0}+1, k-1\right]$ and all $j \in\left[k_{0}+1, p-1\right]$ given by Propositions 8 and 9.

Theorem 13. Consider $\mathbf{S}$ and $\overline{\mathbf{S}}$ given by (1) and $(2)$, respectively. $(\tilde{\mathbf{S}}, \tilde{J}) \supset(\mathbf{S}, J)$ if the matrices $M_{\Pi}, M_{Q^{*}}(k), N_{R^{*}}(k)$ have the following structure, respectively:

$$
\left(\begin{array}{cccc}
0 & M_{\Pi_{12}} & -M_{n_{12}} & 0 \\
M_{\Pi_{12}}^{T}-M_{\Pi_{23}}-M_{\Pi_{23}}^{T}-M_{\Pi_{33}} & M_{n_{23}} & M_{\Pi_{24}} \\
-M_{\Pi_{12}}^{T} & M_{\Pi_{23}}^{T} & M_{n_{33}} & -M_{\Pi_{24}} \\
0 & M_{n_{24}}^{T} & -M_{\Pi_{24}}^{T} & 0
\end{array}\right),
$$




$$
\begin{aligned}
& \left(\begin{array}{cccc}
0 & M_{Q_{i 2}} & -M_{Q_{i 2}} & 0 \\
M_{Q_{j 2}}^{T} & -M_{Q_{23}}-M_{Q_{23}}^{T}-M_{Q_{33}^{*}} & M_{Q_{23}^{*}} & M_{Q_{24}^{*}} \\
-M_{Q_{12}}^{T} & M_{Q_{23}}^{T} & M_{Q_{33}^{*}} & -M_{Q_{24}^{*}} \\
0 & M_{Q_{24}}^{T} & -M_{Q_{24}}^{T} & 0
\end{array}\right)(k), \\
& \left(\begin{array}{cccc}
0 & N_{R_{12}^{*}} & -N_{R_{12}^{*}} & 0 \\
N_{R_{i 2}^{*}}^{T}-N_{R_{23}^{*}}-N_{R_{23}^{*}}^{T}-N_{R_{33}^{*}} & N_{R_{23}^{*}} & N_{R_{24}^{*}} \\
-N_{R_{i 2}^{*}}^{T} & N_{R_{23}^{*}}^{T} & N_{R_{33}^{*}}^{T} & -N_{R_{24}^{*}}^{T} \\
0 & N_{R_{24}^{*}}^{T} & -N_{R_{24}^{*}}^{T} & 0
\end{array}\right)(k),
\end{aligned}
$$

and either

$$
\begin{aligned}
& \text { a) } M(p)=\left(\begin{array}{cccc}
0 & 0 & 0 & 0 \\
M_{21} & M_{22} & M_{23} & M_{24} \\
-M_{21} & -M_{22} & -M_{23} & -M_{24} \\
0 & 0 & 0 & 0
\end{array}\right)(p) \text {, } \\
& N(p)=\left(\begin{array}{cccc}
0 & N_{12} & -N_{12} & 0 \\
N_{21} & N_{22} & N_{23} & N_{24} \\
-N_{21} & -\left(N_{22}+N_{23}+N_{33}\right) & N_{33} & -N_{24} \\
0 & N_{42} & -N_{42} & 0
\end{array}\right)(p)
\end{aligned}
$$

or

$$
\text { b) } \begin{aligned}
M(p) & =\left(\begin{array}{llll}
0 & M_{12} & -M_{12} & 0 \\
0 & M_{22} & -M_{22} & 0 \\
0 & M_{32} & -M_{32} & 0 \\
0 & M_{42} & -M_{42} & 0
\end{array}\right)(p), \\
N(p) & =\left(\begin{array}{llll}
0 & N_{12} & -N_{12} & 0 \\
0 & N_{22} & -N_{22} & 0 \\
0 & N_{32} & -N_{32} & 0 \\
0 & N_{42} & -N_{42} & 0
\end{array}\right)(p)
\end{aligned}
$$

hold for all fixed $k \in\left[k_{0}, k_{f}\right]$ and all $p \in\left[k_{0}+\right.$ $1, k-1]$,

Theorem 14. Consider $\mathbf{S}$ and $\overline{\mathbf{S}}$ given by (1) and (2) satisfying $\tilde{\mathbf{S}} \supset \mathbf{S}$, respectively. A control law $\bar{u}(k)=-\bar{K}(k) \bar{x}(k)$ for $\tilde{\mathbf{S}}$ is contractible to the control law $u(k)=-K(k) x(k)$ of $\mathrm{S}$ if $F(k)=$

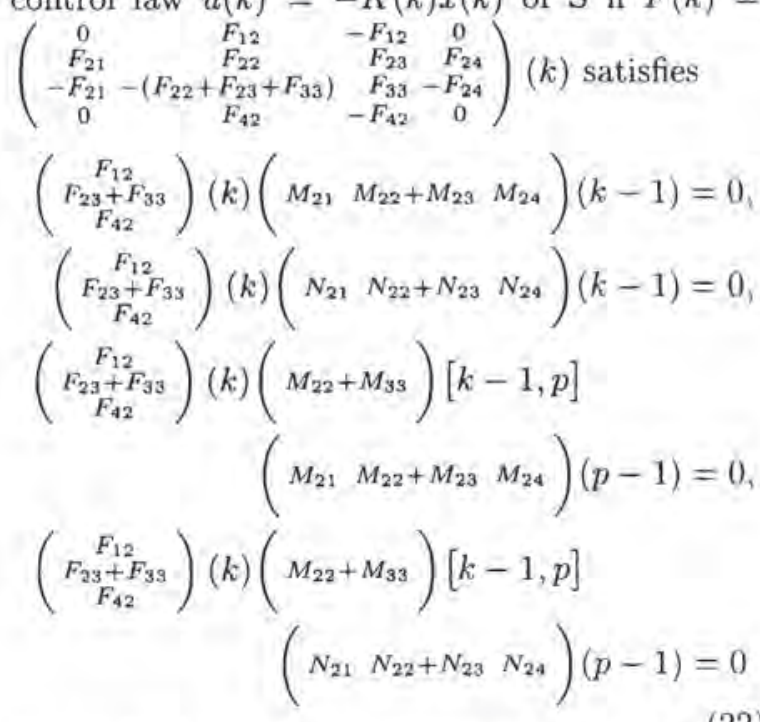

for all fixed $k \in\left[k_{0}, k_{\ell}\right]$ and all $p \in\left[k_{0}+1, k-1\right]$.

Proof. Consider $\bar{F}(k)=T_{B}^{-1} F(k) T_{A}$ with $T_{B}^{-1}, T_{A}$ given by $(19) . \hat{F}_{11}(k)=0$ and the other matrix blocks $\bar{F}_{i j}(k), i, j=1,2$ can be identified from Theorem 11. Thus, we obtain the structure of $F(k)$. Further, we get (22) by imposing (17).

Remark. Choosing $F_{12}(k)=0,\left(F_{23}+F_{33}\right)(k)=$ 0 and $F_{42}(k)=0$ for all $k \in\left[k_{\mathrm{a}}, k_{\mathrm{f}}\right]$ by Theorem 14 , then any control law designed for $\tilde{\mathrm{S}}$ is contractible to $\mathbf{S}$. Moreover, we may identify two important cases from (21) as follows:

$$
\begin{aligned}
& \text { a) } M_{12}(p)=0,\left(M_{23}+M_{33}\right)(p)=0, M_{42}(p)=0, \\
& \text { b) } M_{21}(p-1)=0, \quad\left(M_{22}+M_{23}\right)(p-1)=0, \\
& M_{24}(p-1)=0, \quad N_{21}(p-1)=0
\end{aligned}
$$

for all fixed $k \in\left[k_{n}, k_{t}\right]$ and all $p \in\left[k_{0}+1, k-1\right]$.

\section{CONCLUSION}

The inclusion principle has been specialized for the LQ control design for discrete-time LTV systems. The strategy of generalized selection of complementary matrices has been developed. Explicit conditions on their structure have been proved, including conditions on contractibility of controllers. A systematic procedure for the selection of complementary matrices is included for a particular set of expansion/contraction matrices.

\section{REFERENCES}

Bakule, L., J. Rodellar and J.M. Rossell (2000a). Generalized selection of complementary matrices in the inclusion principle. IEEE Transactions on Automatic Control AC45(6), 1237-1243.

Bakule, L., J. Rodellar and J.M. Rossell (2000b). Structure of expansion-contraction matrices in the inclusion principle for dynamic systems. SIAM Journal on Matrix Analysis and Applications 21(4), 1136-1155.

Ikeda, M., D.D. Siljak and D.E. White (1981). Decentralized control with overlapping information sets. Journal of Optimization Theory and Applications 34(2), 279-310.

Ikeda, M., D.D. Siljak and D.E. White (1984a). An inclusion principle for dynamic systems. IEEE Transactions on Automatic Control AC-29(3), 244-249.

Ikeda, M., D.D. Siljak and D.E. White (1984b). Overlapping decentralized control of linear time-varying systems. In: Advances in Large Scale Systems. Vol. 1. pp. 93-116. JAI Press. Greenwich, Connecticut.

Ito, H. (1998). Overlapping decomposition for multirate decentralized control. In: Preprints of the 8th IFAC/IFORS/IMACS/IFIP Symposium on Large Scale Systems: Theory and Applications, Patras. Vol. 1. pp. 259-264.

Rossell, J.M. (1998). Contribution to decentralized control of large-scale systems via overlapping models. PhD thesis. Technical University of Catalonia, Barcelona, Spain. (In Spanish).

Rugh, W.J. (1996). Linear System Theory (2nd ed). Prentice Hall. Upper Saddle River, New Jersey.

Siljak, D.D. (1991). Decentralized Control of Complex Systems. Academic Press. New York. 